\title{
SOURCE RECONSTRUCTION VIA MOBILE AGENTS IN SENSOR NETWORKS: THROUGHPUT- DISTORTION CHARACTERISTICS
}

\author{
Min Dong and Lang Tong \\ School of Electrical and Computer Engineering \\ Cornell University, Ithaca, NY 14853 \\ \{mdong,ltong\}@ece.cornell.edu \\ and \\ Brian M. Sadler \\ Army Research Laboratory \\ Adelphi, MD 20783 \\ bsadler@arl.army.mil
}

\begin{abstract}
We consider the problem of reconstructing a signal field measured by a large scale sensor network with mobile agents. Sensors transmit packets containing measurement data to mobile agents using either random or deterministic medium access control (MAC) schemes, and the signal field is reconstructed by mobile agents that minimize the mean square error of the reconstruction. For the one-dimensional GaussMarkov field, we investigate the relation between the system throughput and reconstruction distortion, for different types of MAC schemes. We show that at low throughput level, increasing system throughput decreases the reconstruction distortion considerably. But the improvement is much less when the throughput is relatively high. We also show that the choice of MAC schemes can affect the reconstruction performance significantly, especially when the measurement noise is low.
\end{abstract}

\section{INTRODUCTION}

A reachback sensor network with mobile agents is proposed in [1] as a new network structure for low power, large scale sensor networks. In such network, sensors and mobile agents are two types of operating nodes. Sensors are densely deployed in the field to sense information or take measurements, and mobile agents are in charge of collecting information from sensors. At the time of data collection, sensors transmit their data directly back to the mobile agents. By fully utilizing mobile agents' capability, such sensor network structure simplifies node level processing and can benefit from node redundancy for energy efficient and reliable communications.

This work was supported in part by the Army Research Office under Grant ARO-DAAB19-00-1-0507, the Multidisciplinary University Research Initiative (MURI) under the Office of Naval Research Contract N00014-00-1-0564, and Army Research Laboratory CTA on Communication and Networks under Grant DAAD19-01-2-0011.
One type of applications in such sensor network is to provide measurements of some phenomena of interest in a field, such as temperature. Specifically, the physical source information in a field $A$ can be viewed as a continuous stochastic process. A very large amount of sensors are spread in the field for local measurements. At a prearranged time, each sensor senses the information and stores its measurement. When the mobile agent is ready to collect data, sensors transmit their packets back to the mobile agent according to a specified medium access control (MAC) protocol. After a fixed collecting time, the mobile agent receives some data packets from some locations in the field. Based on these samples, we reconstruct the original signal field.

For sensor networks with random access MAC schemes, due to the access randomness, both the number of data packets successfully received and their origins are random. This appears as if the mobile agent randomly samples (both the location and the number of samples) the measurement data in $A$ during the fixed time duration $T$. Intuitively, the more data samples collected, the better signal reconstruction performance. Notice that the average number of data samples the mobile agent receives is determined by the throughput of the network. Then how does the reconstruction performance vary with the throughput? The significance of characterizing the relation between throughput and reconstruction distortion lies in two folds: first, a given system throughput provides the expected field reconstruction performance under such sensor network; second, and probably more important, to target the reconstruction at a specified distortion tolerance level, it indicates how much throughput the network is required, and thus directs the quality-ofservice (QoS) specific network design. Furthermore, it is also important to know the variation rate of distortion under different throughput levels, as it indicates the effectiveness of throughput improvement on the reconstruction performance. Unlike conventional communication networks 
where independent data are generated from each user nodes, having (highly) correlated data among sensor nodes is an important feature in a densely deployed sensor network. Therefore, another related question to ask is that how different types of MAC schemes affect reconstruction performance? The validity of such question is evident from the fact that the pattern of received data sample locations affects signal reconstruction performance. Intuitively, a uniformly sampled data results in less reconstruction distortion than that from using data samples grouped in a small subarea.

In this paper, we consider the problem of measuring a one-dimensional signal field using a reachback sensor network with a mobile agent. The source signal is modeled as a Gauss-Markov stochastic process. Based on the received data at the mobile agent, the minimum mean square error (MMSE) estimator is used for signal reconstruction. The reconstruction distortion is measured by the maximum estimation error of reconstruction. We investigate the relation between the throughput of the network and reconstruction distortion. We consider both noiseless and noisy measurement cases. We show that increasing throughput decreases the distortion considerably at low throughput level, but this effect is much less when the throughput is relatively high. Besides random access MAC schemes, we also evaluate the performance under the MAC with uniform packet reception, i.e., the packets received are from uniformly spaced locations in the field. Comparing the performance under the above two types of MAC schemes, we show that the latter type of MAC schemes significantly improves reconstruction performance, especially at low measurement noise level. This indicates the importance of sensor-network specific MAC designs on the ultimate system performance.

The problems on sensor network communications have attracted a growing research interest. In terms of medium access control, many MAC protocols have been proposed aiming to the special needs and requirements for both ad hoc sensor networks [2-5] and reachback sensor networks with mobile agents $[6,7]$. The problem of signal estimation and reconstruction, admitting the specific characteristics under such sensor network settings, is still a relatively new topic for investigation. Perhaps the most relevant work is [8], where the problem of random sampling of a dynamic system is discussed, and sequential estimation of a dynamic source is considered using random sampling in time.

\section{PROBLEM STATEMENT}

\subsection{Source Model}

Consider a one-dimensional field of length $D$, denoted by $A=[0, D]$. Let $S_{t}(x)(x \in A)$ be the source of interest in $A$ at time $t$. We assume that the spatial dynamic of $S_{t}(x)$ is a stationary Gaussian random process given by the following linear stochastic differential equation:

$$
d S_{t}(x)=f S_{t}(x) d x+\sigma d W_{t}(x)
$$

where $f, \sigma \in \mathbf{R}$ are known and $f<0^{1},\left\{W_{t}(x): x \geq 0\right\}$ is a standard Brownian motion, and $S_{t}(x) \sim \mathcal{N}\left(0, \frac{\sigma^{2}}{|2 f|}\right)$.

\subsection{Sensor Measurement and Data Reception via Mo- bile Agent}

Suppose that a very large amount of sensors are uniformly deployed in $A$, and every sensor knows its relative location. The sensor measurement at time $t$ is given by

$$
Y_{t}(x)=S_{t}(x)+N_{t}(x)
$$

where $N_{t}(x)$ is an independent and identically distributed (i.i.d.) zero mean white Gaussian measurement noise with variance $\sigma_{n}^{2}$. Each sensor stores its local measurement of signal and waits for the mobile agent to collect. Note that we will reconstruct the source signal $\left\{S_{t}(x): x \in A\right\}$ at time $t$ based only on the sensor measurements at that time: $\left\{Y_{t}(x): x \in A\right\}$. Therefore, we drop the time index for brevity in the following presentation.

When the mobile agent is ready for data collection, sensors transmit packets, consisting measurement data along with its location information, to the mobile agent through a common wireless channel, using either random or deterministic MAC schemes. To remove the boundary effect for signal reconstruction, we assume that the mobile agent always first obtains packets from the two boundaries of $A$, i.e., $Y(0)$ and $Y(D)$, before starting the collection of the rest packets. After some fixed collecting time $T$, assume that the mobile agent receives total $M$ packets originated from some $M$ points in $A$. Based on these data samples, we reconstruct the signal field by smoothing technique using the MMSE estimator. Fig. 1 shows an example of a resulting sampling of the signal field in $A$. Given the received data at points $\mathbf{p}_{M}=\left[0, P_{1}, \cdots, P_{M}, D\right]$, the signal reconstruction by MMSE smoothing is then given by

$$
\hat{S}(x)=E\left\{S(x) \mid Y_{p}, p \in \mathbf{p}_{M}\right\}, \quad x \in A .
$$

If a random access MAC scheme is used in the sensor network, both the number of data packets successfully received $(M)$ and their origins $\left(\mathbf{p}_{M}\right)$ are random. This appears as if the mobile agent randomly samples the sensor measurement data in $A$ (both the location and the number

\footnotetext{
${ }^{1} f<0$, thus, (1) admits a stationary solution.
} 
of samples) during collection time $T$. Let $\lambda$ be the throughput of the network. We assume that the number of received packets $M$ during time $T$ is Poisson distributed with rate $\lambda$ :

$$
P(M=n)=e^{-\lambda T} \frac{(\lambda T)^{n}}{n !} .
$$

Then, the average number of received data samples during $T$ is given by $E\{M\}=\lambda T$.

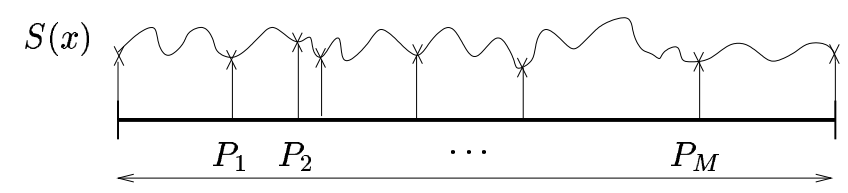

$D$

Fig. 1: a 1-D signal field sampled by a sensor network

\subsection{Source Reconstruction Distortion}

Given $M+2$ received packets from $\mathbf{p}_{M}$, we define the field reconstruction distortion by the maximum estimation error in $A$ :

$$
\mathcal{E}\left(\mathbf{p}_{M}\right) \triangleq \max _{x \in A} E\left\{|\hat{S}(x)-S(x)|^{2} \mid \mathbf{p}_{M}\right\} .
$$

Under throughput $\lambda$ and collecting time $T$, the average distortion is then given by

$$
\begin{aligned}
\overline{\mathcal{E}}(\lambda T) & \triangleq E\left\{\mathcal{E}\left(\mathbf{p}_{M}\right)\right\} \\
& =E\left\{E\left\{\mathcal{E}\left(\mathbf{p}_{M}\right) \mid M\right\}\right\}
\end{aligned}
$$

where in (6), the second expectation is taken over $\mathbf{p}_{M}$ for a given $M$, and the first one is taken over $\mathrm{M}$.

Clearly, the average distortion $\overline{\mathcal{E}}(\lambda T)$ is a function of the throughput $\lambda$ of this sensor network. Intuitively, the network with higher throughput results in, on average, more data samples received at the mobile agent, which provides better estimate of the signal field and results in lower reconstruction distortion. In the following, we analyze how the reconstruction distortion $\overline{\mathcal{E}}(\lambda T)$ varies with the throughput $\lambda$.

\section{SOURCE RECONSTRUCTION: DISTORTION VS. THROUGHPUT}

We assume that under the random access MAC scheme, the data packet generated from any sensor has equal chance to be successively received by the mobile agent. Therefore, given there are $M$ packets received ${ }^{2}$, the packet generation

\footnotetext{
${ }^{2}$ The actual number of received packets is $M+2$. For convenience, we only count those packets not from the two boundaries of $A$.
}

locations $\left\{P_{1}, \cdots, P_{M}\right\}$ are i.i.d. random variables with uniform distribution $U(0, D)$.

To contrast with such random access MAC schemes, we also consider another type of MAC schemes, referred to as the deterministic MAC with uniform reception. This type of MAC schemes is designed in such a way that, instead of their generation locations being random, the $M$ received packets at the mobile agent are originated from uniformly spaced locations in $A$, i.e., $\mathbf{p}_{M}=\left\{0, \frac{D}{M+1}, \frac{2 D}{M+1}, \cdots, D\right\}$.

In the following, we evaluate the average distortion under both types of MAC schemes, and compare their performance.

\subsection{Sensors with Noiseless Measurement Data}

We first consider the case when sensor measurements contain no noise, i.e., $Y(x)=S(x)$. Given $M=m$ received packets, the corresponding order statistics of the packet generation locations $\left\{P_{1}, \cdots, P_{m}\right\}$, denoted by $P_{(1)}<\cdots<$ $P_{(m)}$, have the joint density given by

$f_{P_{(1)}, \cdots, P_{(m)}}\left(x_{1}, \cdots, x_{m}\right)= \begin{cases}\frac{m !}{D^{m}}, & \text { if } 0<x_{1}<\cdots<x_{m}<D \\ 0, & \text { otherwise. }\end{cases}$

From (1), due to the Markov property of the stochastic process, given samples at locations $p_{(i)}$ and $p_{(i+1)}$, the signal $S(x)$, where $p_{(i)}<x<p_{(i+1)}$, is completed determined by $S\left(p_{(i)}\right)$ and $S\left(p_{(i+1)}\right)$. By the Gaussian property of the process $\{S(x)\}$, the MMSE estimator in (3) is then given by

$$
\hat{S}(x)=E\left\{S(x) \mathbf{S}_{p}^{(i)}{ }^{H}\right\} E^{-1}\left\{\mathbf{S}_{p}^{(i)} \mathbf{S}_{p}^{(i)}{ }^{H}\right\} \mathbf{S}_{p}^{(i)}
$$

for $p_{(i)}<x<p_{(i+1)}$, where $\mathbf{S}_{p}^{(i)}=\left[S\left(p_{(i)}\right), S\left(p_{(i+1)}\right)\right]^{T}$. Let

$$
d_{i} \triangleq P_{(i+1)}-P_{(i)}
$$

for $i=0, \cdots, M .^{3}$ Then, the corresponding maximum MMSE of $\hat{S}(x)$, for $p_{(i)}<x<p_{(i+1)}$, is obtained at the middle point of $p_{(i)}$ and $p_{(i+1)}$, and it is given by

$$
\begin{aligned}
& \max _{p_{(i)}<x<p_{(i+1)}} E\left\{|\hat{S}(x)-S(x)|^{2} \mid P_{(i)}, P_{(i+1)}\right\} \\
& =\max _{p_{(i)}<x<p_{(i+1)}}\left\{E|S(x)|^{2}-\right. \\
& \left.\quad E\left\{S(x) \mathbf{S}_{p}^{(i)}{ }^{H}\right\} E^{-1}\left\{\mathbf{S}_{p}^{(i)} \mathbf{S}_{p}^{(i)}{ }^{H}\right\} E^{H}\left\{S(x) \mathbf{S}_{p}^{(i)}{ }^{H}\right\}\right\} \\
& =\frac{1-e^{f d_{i}}}{1+e^{f d_{i}}} \frac{\sigma^{2}}{|2 f|}
\end{aligned}
$$

Therefore, the distortion $\mathcal{E}\left(\mathbf{p}_{M}\right)$ in (4) is determined by the maximum of distances between any two consecutive data

\footnotetext{
${ }^{3}$ we denote $P_{(0)}=0$ and $P_{(M+1)}=D$.
} 
samples

$$
\mathcal{E}\left(d_{\max }\right)=\mathcal{E}\left(\mathbf{p}_{M}\right)=\frac{1-e^{f d_{\max }}}{1+e^{f d_{\max }}} \frac{\sigma^{2}}{|2 f|}
$$

where

$$
d_{\max }=\max _{0 \leq i \leq M} d_{i}
$$

Thus, to calculate the average distortion $\overline{\mathcal{E}}(\lambda T)$, we only need to find the probability distribution of the maximum sample distance.

Under the random access MAC schemes, given $M=$ $m$, we now find the cdf of the maximum sample distance $F_{d_{\max }}(\cdot \mid m)$. By the transformation of random variables, from (7) and (9), we have the joint distribution of sample distances $\left\{d_{0}, \cdots, d_{m-1}\right\}$ given by

$f_{d_{0}, \cdots, d_{m-1}}\left(x_{0}, \cdots, x_{m-1}\right)= \begin{cases}\frac{m !}{D^{m}}, & \text { if } x_{i} \geq 0, \sum_{i=0}^{m-1} x_{i} \leq D \\ 0, & \text { otherwise }\end{cases}$

and

$$
\begin{aligned}
F_{d_{\max }}(x \mid m) & =\operatorname{Pr}\left(d_{0} \leq x, \cdots, d_{m-1} \leq x, D-\sum_{i=0}^{m-1} d_{i} \leq x\right) \\
& =\int_{0}^{x} \cdots \int_{0}^{x} g\left(x_{0}, \cdots, x_{m-1}\right) d x_{0} \cdots d x_{m-1}(12)
\end{aligned}
$$

where

$g\left(x_{0}, \cdots, x_{m-1}\right)$

$=f_{d_{0}, \cdots, d_{m-1}}\left(x_{0}, \cdots, x_{m-1}\right) \mathbf{1}_{\left[D-\sum_{i=0}^{m-1} x_{i} \leq x\right]}\left(x_{0}, \cdots, x_{m-1}\right)$

and $\mathbf{1}_{B}(x)$ is the indicator function

$$
\mathbf{1}_{B}(x)= \begin{cases}1, & \text { if } x \in B \\ 0, & \text { if } x \notin B .\end{cases}
$$

Notice that to calculate $F_{d_{\max }}(x \mid m)$ in (12) is essentially to calculate the volume of a $m$-dimensional hypercube under the two hyperplane constraints. The resulting formula for $F_{d_{\max }}(x \mid m)$ is given in (17). Thus, the average distortion of signal reconstruction $\overline{\mathcal{E}}(\lambda T)$ in (6) is obtained by

$$
\begin{aligned}
& \overline{\mathcal{E}}(\lambda T)=\sum_{m=0}^{\infty}\left(\int_{0}^{\infty} P\left(\mathcal{E}\left(d_{\max }\right)>x\right) d x\right) e^{-\lambda T} \frac{(\lambda T)^{m}}{m !} \\
&=e^{-\lambda T} \sum_{m=0}^{\infty}[ \int_{\mathcal{E}\left(\frac{D}{m+1}\right)}^{\mathcal{E}(D)}\left(1-F_{d_{\max }}\left(\mathcal{E}^{-1}(x) \mid m\right)\right) d x \\
&\left.+\mathcal{E}\left(\frac{D}{m+1}\right)\right] \frac{(\lambda T)^{m}}{m !} .
\end{aligned}
$$

For deterministic MAC with uniform reception, $\overline{\mathcal{E}}(\lambda T)$ can be readily obtained as follow

$$
\overline{\mathcal{E}}^{\mathrm{eq}}(\lambda T)=e^{-\lambda T} \frac{\sigma^{2}}{|2 f|} \sum_{m=0}^{\infty}\left(\frac{1-e^{f \frac{D}{m+1}}}{1+e^{f \frac{D}{m+1}}}\right) \frac{(\lambda T)^{m}}{m !} .
$$

Fig. 2 shows the curves of throughput-distortion characteristics under the two types of MAC schemes. We set $f=-0.1, \sigma^{2}=0.2$, and $T=100$. We observe that when $\lambda$ is low, the average distortion decreases fast with increasing throughput $\lambda$. However, as $\lambda$ becomes larger, the decreasing rate of distortion becomes slower. In other word, the signal reconstruction performance is more sensitive to the throughput improvement at low throughput level. Comparing the performance under random MAC schemes with that under the deterministic MAC scheme with uniform reception, we observe that a significant performance gain can be achieved using the latter scheme. This indicates that, in such sensor networks with highly correlated data information, the MAC design with uniform reception is highly desired.

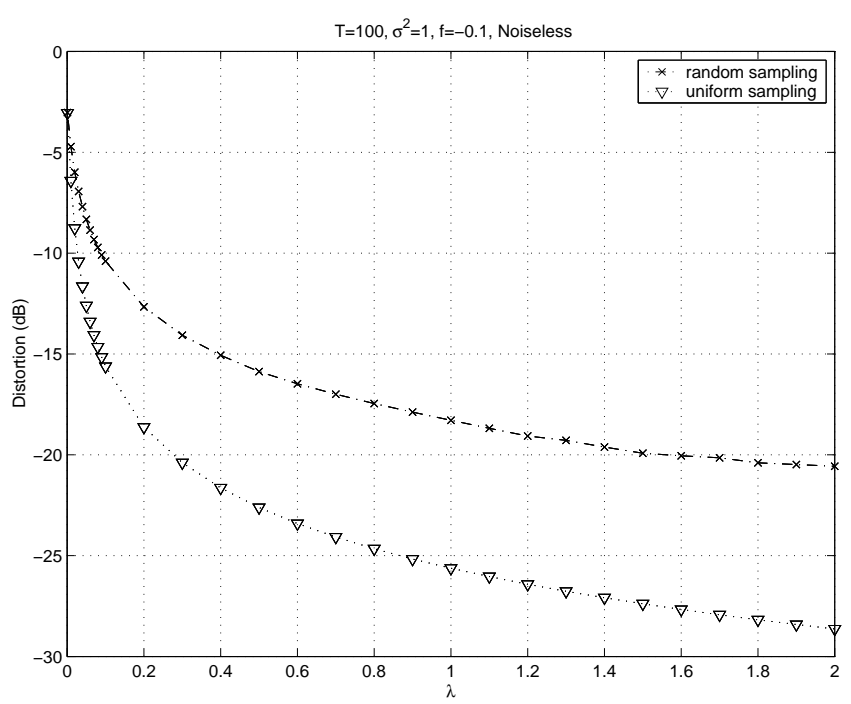

Fig. 2: Distortion vs. throughput

\subsection{Sensors with Noisy Measurement Data}

When noises are presented in the signal measurements, all received data samples need to be used to reconstruct signal at each point in $A$. Let $\mathbf{S}_{p_{M}}=\left[S\left(P_{(0)}\right), \cdots, S\left(P_{(M)}\right)\right]^{T}$ be the source signal of which the noisy measurements are received at the mobile agent. In this case, the MMSE of $\hat{S}(x)$ is given by

$$
\begin{aligned}
\mathcal{M}\left(x ; \mathbf{p}_{M}\right) & \triangleq E\left\{|\hat{S}(x)-S(x)|^{2} \mid \mathbf{p}_{M}\right\} \\
& =\frac{\sigma^{2}}{|2 f|}-\mathbf{r}\left(x, \mathbf{p}_{M}\right)\left(\mathbf{R}_{\mathbf{p}_{M}}+\sigma_{n}^{2} \mathbf{I}\right)^{-1} \mathbf{r}^{H}\left(x, \mathbf{p}_{M}\right)
\end{aligned}
$$




$$
F_{d_{\max }}(x \mid m)= \begin{cases}0 & \text { if } 0 \leq x<\frac{D}{m+1} \\
\sum_{i=0}^{k}(-1)^{i}\left(\begin{array}{c}
m+1 \\
i
\end{array}\right)\left[(m-i+1) \frac{x}{D}-1\right]^{m} & \text { if } \frac{D}{m-k+1} \leq x<\frac{D}{m-k}, \text { for } k=0, \cdots, m-2 \\
1-(m+1)\left(1-\frac{x}{D}\right)^{m} & \text { if } \frac{D}{2} \leq x \leq D\end{cases}
$$

where $\mathbf{r}\left(x, \mathbf{p}_{M}\right) \triangleq E\left\{S(x) \mathbf{S}_{p_{M}}^{H}\right\}$ and $\mathbf{R}_{\mathbf{p}_{M}} \triangleq E\left\{\mathbf{S}_{p_{M}} \mathbf{S}_{p_{M}}^{H}\right\}$. Hence, $\overline{\mathcal{E}}(\lambda T)$ is obtained as

$$
\begin{gathered}
\overline{\mathcal{E}}(\lambda T)=e^{-\lambda T} \sum_{m=0}^{\infty}\left(\int_{0}^{D} \cdots \int_{0}^{D} \max _{y} \mathcal{M}\left(y ; \mathbf{x}_{m}\right)\right. \\
\left.f_{P_{(1)}, \cdots, P_{(m)}}\left(x_{1}, \cdots, x_{m}\right) d x_{1} \cdots d x_{m}\right) \frac{(\lambda T)^{m}}{m !} .
\end{gathered}
$$

The distortion under the MAC with uniform reception can be obtained similarly

$$
\overline{\mathcal{E}}^{\mathrm{eq}}(\lambda T)=e^{-\lambda T} \sum_{m=0}^{\infty} \mathcal{M}\left(x ; \mathbf{p}_{m}\right) \frac{(\lambda T)^{m}}{m !}
$$

where in this case $\mathbf{p}_{m}=\left[0, \frac{D}{m+1}, \cdots, D\right]$.

Fig. 3 shows the distortion curve under the variation of throughput at $S N R=10 \mathrm{~dB}$ and $20 \mathrm{~dB}$, respectively. The same setup as in Fig. 2 is used. We observe that as the noise becomes higher, the curve become more flat. This indicates that due to the measurement noise, throughput improvement is less effective on the reconstruction performance gain. Furthermore, we observe that the performance gap under the two different MAC schemes becomes smaller as the measurement noise become higher.

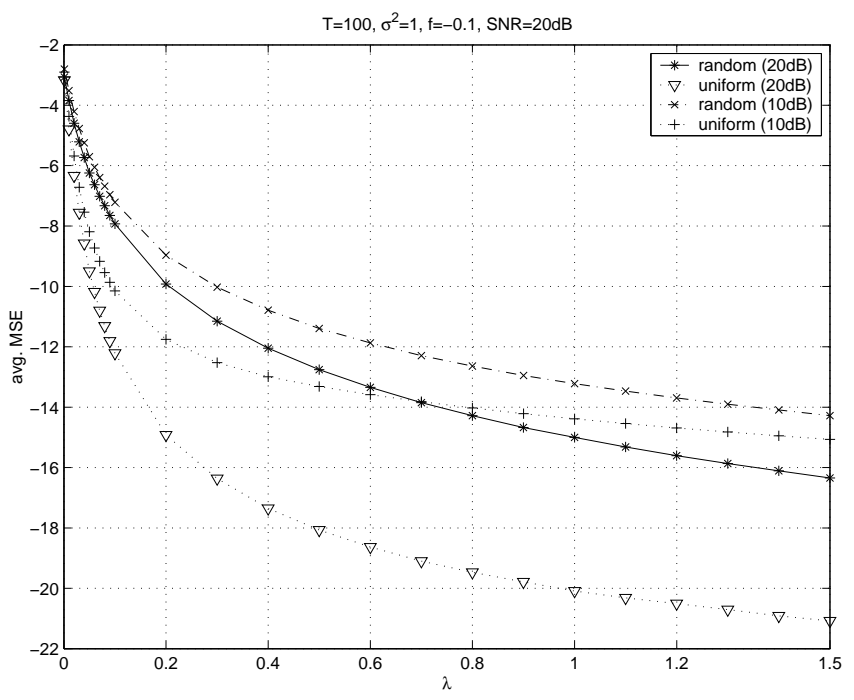

Fig. 3: Distortion vs. throughput

\section{CONCLUSION}

In this paper, the problem of reconstructing a signal field measured by a densely deployed sensor network with a mobile agent is considered. We investigate the relation between source reconstruction performance and network throughput under both random and deterministic MAC designs. We show that throughput improvement is more effective on reconstruction performance gain at a low throughput level than that at a relatively high level. Furthermore, we show that designing a MAC scheme with uniform reception can obtain a considerable gain on reconstruction performance.

\section{REFERENCES}

[1] L. Tong, Q. Zhao, and S. Adireddy, "Sensor networks with mobile agents," in Proc. IEEE MILCOM'03, Boston, MA, October 2003.

[2] R. Iyer and L. Kleinrock, "QoS Control for Sensor Networks," in Proc. ICC 2003, May 2003.

[3] K. Sohrabi, J. Gao, V. Ailawadhi, and G. Pottie, "Protocols for self-organization of a wireless sensor network," IEEE Personal Communication, pages 16-27, October 2000.

[4] A. Woo and D. Culler, "A transmission control scheme for medium access in sensor networks," in Proc. ACM/IEEE Conference on Mobile Communication and Networking, pages 221-235, Rome, Italy, July 2001.

[5] W. Ye, J. Heidemann, and D. Estrin, "An energy-efficient MAC protocols for wireless sensor networks," in Proc. of IEEE INFOCOM, pages 1567-1576, New York, June 2002.

[6] S. Adireddy, P. Venkitasubramaniam, and L. Tong, "Opportunistic ALOHA and cross layer design for sensor networks," in Proc. IEEE MILCOM'03, Boston, MA, October 2003.

[7] Q. Zhao and L. Tong, "QoS specific information retrieval for densely deployed sensor network," in Proc. IEEE MILCOM'03, October 2003.

[8] M. Micheli and M. I. Jordan, "Random Sampling of a Continuous-time Stochastic Dynamical System," Proc. of the 15th International Symposium on the Mathematical Theory of Networks and Systems (MTNS 2002), University of Notre Dame, South Bend, Indiana, August 2002. 